\title{
Multidisciplinary cancer care: we need to practice what we preach
}

\author{
Josep M. Borràs · Tàrsila Ferro
}

$\mathrm{O}$ ne of the key recommendations of the Spanish Cancer Strategy in Cancer is to implement multidisciplinary cancer care as the organisational model for clinical decision making on diagnosing and delivery of cancer treatment. This recommendation is also considered to be a contribution to reducing the clinical variability in cancer as well as the inequity of access to diagnosis and therapy [1]. This approach is consistent with what has been set as the goals of the European policy in cancer care [2].

It is not very difficult to explain why this approach is needed: the number of professionals from different specialties involved in diagnosing and treating a cancer patient; the patient's journey through the health care system that involves different departments and hospitals; the variabity in the perspectives that professionals could have when coping with the therapeutic decision-making process, which need to be discussed and agreed upon before beginning therapy; and the relevance of coordinating the entire process in a sequential and logical pathway, which should be communicated and shared with the patient in an understandable way. On the other hand, the levels of clinical complexity surrounding cancer patients, which is increasing due to factors such as co-morbidity associated with aging and the progressive introduction of patient preferences in the

J.M. Borràs ( $(\mathbb{})$

Scientific Coordinator

Spanish Cancer Strategy

Ministry of Health and Social Policy

Hospital Duran i Reynals

Gran Via, s/n - km 2,7

ES-08907 L'Hospitalet de Llobregat, Barcelona, Spain

e-mail: jmborras@iconcologia.net

T. Ferro

Coordinator, Catalan Cancer Plan

School of Nursing

University of Barcelona

L'Hospitalet de Llobregat, Barcelona, Spain

decision-making process, implies a trend towards physician specialisation by tumour site. This trend has, in turn, made the need for building a forum for all professionals from different specialties to assure a global and shared view of the patient. This is precisely what multidisciplinary care intends to fulfil.

A multidisciplinary cancer team has been defined as professionals of different health care disciplines who meet as a group at a given time (whether physically in one place or by video- or teleconferencing) to discuss a given patient and who are able of contributing independently to the diagnostic and treatment decisions about the patient [3]. The team composition is dependent on the tumour site, which should include specialists in the diagnostic area (pathology, nuclear medicine, radiology) in the therapeutic area (surgeons, medical oncologists, radiation oncologists, other specialties) nurses, psychooncologists, palliative care physicians, and others, according to each hospital and locally available resources. The organization will depend on the local particularities, but some aspects are very relevant, such as the decision to review all cases of the specific site, participation of all involved specialists -which is not so easy in some cases (for instance, the need for a radiation oncologist when there are no radiotherapy facilities in the hospital)- establish a single point of entry for patients to the hospital, making decisions that are binding on the team and communication quality among participating professionals [4].

So, what about the effectiveness of these multidisciplinary teams (MDT) in cancer? In a review of the literature, it was found that MDT improves patient outcomes, including survival, as well as patient and professional satisfaction [5].

The question is, then: Why it is this team approach not so extended in clinical practice? Several barriers limit the development of this approach to cancer care. First are the separate roles and defensive strategies put in place by the different specialties, which result in a lack of coordination among different professionals and which are also perceived by the patient [6]. Second is the lack of specialists in community hospitals and lack of a coordinator for the MDT and medicolegal concerns in case of disagreement [7]. It 
should be acknowledged that MDT in university hospitals implies a change in the way services are organized: there is a concentration of knowledge based on tumour site specialisation of the professionals involved in MDT and a decentralisation of power from the traditional organ-based medical departments. In fact, the development of MDT in Spain is not homogeneous and shows remarkable differences among hospitals.

There is a clear need for professional involvement and support to this approach to cancer care. In this sense, the institutional declaration published in this issue of Clinical and Translation Oncology and signed by 15 scientific societies (ranging from the diagnosis of cancer to therapeutic and/or palliative care, including the nursing oncology and psycho-oncology societies) and patient associations, is a highly relevant indication of support for MDT in Spain. The commitments of these societies to cooperate in boosting multidisciplinary care in our country, with a focus on management and organizational changes aimed at responding to the complexity of cancer care, fostering quality of care and evaluating outcomes, should be emphasised.

One point deserves special attention, namely, recognition of the multiple MDT models that could be applied in a particular hospital and local agreements that should be es- tablished among the involved professionals. However, several requirements should always be fulfilled: specialisation by disease according to the volume and complexity of cases of the hospital; presence of a coordination role assumed by a professional; and use of best evidence for clinical practice. These requirements are the core of implementing the MDT in a hospital, along with promoting good cooperation and communication among all involved professionals in order to meet patient needs [6]. Also, the relevance of the coordination with primary health care mentioned in the declaration is very relevant because of the role they could play in patient diagnosis and long-term follow-up, as well as incorporating nursing staff to improve the patient's cancer journey, care, and needs fulfilment.

With this declaration, the scientific societies and patient associations in Spain are aligned with similar position statements made by their American and European counterparts. Moreover, it reinforces the strategic objectives of the Spanish Cancer Strategy of our National Health Service with the aim of reducing the variability in clinical practice that is not attributable to the specific clinical characteristics of the patient. It also strengthens our aim of improving equity of access to a high quality of cancer care throughout Spain, marking a milestone along our path to increased survival and quality of life of cancer patients.

\section{References}

1. Agencia de Calidad y Cohesión del Sistema Nacional de Salud (2010) Estrategia en Cáncer del SNS. Madrid: Ministerio de Sanidad y Política Social

2. Gouveia J, Coleman MP, Haward R et al (2008) Improving cancer control in the European Union: conclusion from the Lisbon roundtable under the
Portuguese EU presidency. Eur J Cancer 44:14571462

3. Fleissig A, Jenkins V, Catt S, Fallowfield L (2006) Multidisciplinary teams in cancer care: are they effective in the UK? Lancet Oncol 7:935-943

4. Molyneux J (2001) Interprofessional teamworking: what makes teams work well? J Interprofesional Care 15:29-35

5. Wright FC, de Vito C, Langer B, Hunter A (2007) Multidisciplinary cancer conferences: a systematic review and development of practice standards. Eur J Cancer 43:1002-1010

6. Committee of Quality Health Care in America, Institute of Medicine (2001) Crossing the quality chasm: a new health system for the 21 st century. Washington, National Academy Press

7. Sidham MA, Paulsen MG (2006) Multidisciplinary care in oncology: medicolegal implications of group decisions. Lancet Oncol 7:951954 